\title{
Structure of Manifolds with Positive Curvature Based on Geometric Analysis
}

\author{
by Shing-Tung Yau \\ Department of Mathematics, Harvard University
}

The Gauss-Bonnet theorem and the Cohn-Vossen inequality show that the only complete surface with positive curvature is either the sphere, $\mathbb{R} \mathbb{P}^{2}$, or the plane.

In higher dimension, the curvature tensor is far more complicated. There are several commonly used partial components of the curvature tensor that had been studied for the whole century. The simplest one is the scalar curvature which is the average of all curvatures at one point. It appeared in the Hilbert action for general relativity. This was studied extensively in connection with general relativity.

\section{Manifolds with Positive Scalar Curvature}

The basic technology to understand spaces with positive scalar curvature consist of the following topics:

1. Conformal deformation under the name of Yamabe problem $[34,35]$. The Yamabe constant, which is obtained by minimizing the total scalar curvature in the conformal class of the metric with a fixed volume, is a conformal invariant. If it is nonnegative, the metric is conformal to a metric with nonnegative scalar curvature.

2. The theory of Dirac equation in conjunction with the Lichnerowicz vanishing theorem [22] which states that non-zero harmonic spinor does not exist for compact spin manifolds with a metric of positive scalar curvature. Hitchin [17] observed how to use the vanishing theorem of Lichnerowicz to prove that the $\alpha$ invariant vanishes for $8 k+1$ and $8 k+2$ spin manifolds admitting a metric of positive scalar curvature from the Atiyah-Singer mod 2 index theorem. In 1974, Lawson-Yau [20] proved that manifolds with effective compact continuous nonabelian group action must admit metrics with positive scalar curvature: hence the vanishing of the above KO characteristic numbers.

3 . The theory of minimal hypersurfaces based on the second variational formula. The basic observation is that: if the ambient manifold admits a metric with positive scalar curvature, then the induced metric on a stable minimal hypersurface can be conformally deformed to one with positive scalar curvature. The strategy is to first use information from topology to construct stable minimal hypersurfaces and then to apply the above observation to do induction on dimensions. When the dimension is reduced to two, we know the problem is settled by Gauss-Bonnet theorem. This theory was due to Schoen and Yau [31], where they also used it to prove the existence of black hole if there is enough matter concentrated in a region with fixed size. The existence of stable minimal hypersurface can be used to find topological obstruction to existence of metrics with positive scalar curvature when dimension is not greater than seven. The dimension restriction comes from regularity of minimal hypersurface. Schoen and I believe that we should be able to remove the dimension constraint now.

4. Schoen-Yau [40] made the important observation that, by conformal deformation technology, it is possible to perform surgery on spheres with trivial normal bundle and codimension $\geq 3$ in a manifold with positive scalar curvature so that the resulting manifold still admits a metric with positive scalar curvature. A year later, Gromov-Lawson [41] reproved the same result using a more direct construction. Some of the key formula was found to be wrong and was claimed to be corrected in [39], except that another authors [36] claimed to find mistakes again. In any case, the Schoen-Yau surgery result reduces the problem of existence of metrics with positive scalar curvature to spin cobordism as was carried out by GromovLawson, Stolz [47] and others.

5. Gromov-Lawson [11] extended the work of Lichnerowicz vanishing theorem [22] to those manifolds which admit covering space that is expanding (a terminology introduced in their paper). Hence they proved that compact manifolds with nonpositive sectional curvature cannot admit metrics with positive scalar curvature. (For dimension $\leq 7$, the result was first proved by Schoen-Yau mentioned in the above paragraph.) It was conjectured by Schoen-Yau that if besides the fundamental group, all higher homotopy groups of a manifold vanish, then the manifold does not admit a metric with positive scalar curvature. Schoen-Yau [30] proved this conjecture if the manifold has dimension not greater than 4.

6. Marques [48] proved that the space of metrics with positive scalar curvature is connected for a three dimensional manifold, while Hitchin [49] proved that metrics with positive scalar curvature is not connected in seven dimension, based on the diffeomorphism group of the seven sphere is not standard. 
7. Connes [50] used the method of noncommutative geometry to derive a topological obstruction for a manifold to have a spin foliation with leaves of positive scalar curvature. It is an interesting question whether such a manifold must itself admit a metric of positive scalar curvature.

8. In the paper by Witten-Yau [57], they studied the question of existence of metrics with positive scalar curvature on a compact manifold which is a conformal boundary of an asymptotically hyperbolic complete Einstein manifold. They proved that the manifold is connected. Based on this result and the surgery result of Schoen-Yau, Yau proposed the conjecture in [51] that it is possible to do surgery on such class of manifolds. Yau also asserted that it is easy to do connected sum of such manifolds. The result was communicated by Daniel Pollack to Mazzeo who wrote a paper with Pacard in [55].

\section{Manifolds with Positive Ricci Curvature}

The variation of the Hilbert action gives rise to the Einstein equation, where the Ricci curvature shows up. The theory of Ricci curvature is important in relation to the theory of Einstein metric. After modding out choice of gauge, this is a deterministic system where the number of unknown variables is the same as number of equations. Manifolds with positive Ricci curvature are manifolds with positive scalar curvature. Hence it encounters similar topological obstructions. However, the splitting theorem of Cheeger-Gromoll shows that compact manifold with nonnegative Ricci curvature has a finite cover given by the product of the torus with a simply connected compact manifold. Hence the fundamental group of such manifold is rather restrictive. One can construct complete manifolds with positive Ricci curvature by taking bundles over other manifolds with positive Ricci curvature. This was carried out by John Nash in [54]. Mckenzie Wang, and Wolfgang Ziller [52] and others. Sha and Yang [53] constructed metrics with positive Ricci curvature on the connected sums of products of spheres and complex projective spaces.

However, Kähler geometry provides the richest class of metrics with positive Ricci curvature.

The following important consequence of the Calabi conjecture is related to metrics with positive Ricci curvature. Given any closed (1.1) form which represents the first Chern form on a Kähler manifold, it can be represented as Ricci curvature form of some Kähler metric. Hence if the first Chern form is positive, we have Kähler manifolds with positive Ricci curvature. The noncompact version of the Calabi conjecture was studied by Yau and announced in the Helsinki congress in 1978. Some detail was written up with Tian in $[45,46]$ to non-compact Kähler manifolds. One can also construct a large set of example of orbifolds with positive Ricci curvature. Bundles over such orbifolds may become nonsingular manifolds with positive Ricci curvature.

\section{Manifolds with Quarter-Pinched Positive Sectional Curvature, Manifolds with Positive Curvature Operator, Manifolds with Isotropic Positive Curvature and Kähler Manifolds with Positive Bisectional Curvature}

The full curvature tensor enables us to define different inequalities for various curvature components. First of all, we know that on the Grassmannian of two-planes in the tangent space at any point of the manifold, we can associate a curvature function called sectional curvature. It is observed by Killing in 1891 and by H. Hopf in 1926 that if this function is constant, the manifold is covered by the round sphere, the Euclidean space, or the hyperbolic space.

Hopf and later H. E. Rauch were interested in a suitable stability statement: when the sectional curvature is close to be a constant, does the manifold resemble a space form?

Rauch was a student of Bochner whose idea of vanishing theorem led to the work of Lichnerowicz on vanishing of harmonic spinors on metrics with positive scalar curvature.

Rauch developed the comparison theorems while Toponogov [37] proved his triangle inequalities. These provided powerful tools to make definite progress on the problem of Hopf. In 1951, Rauch [28] proved that if the sectional curvature is between a constant around 0.75 and 1 , the manifold is homeomorphic to the sphere if it is simply connected.

Klingenberg in 1961 [19] finally proved that if the sectional curvature is greater than $1 / 4$ and not greater than 1 , the manifold is homeomorphic to the sphere if it is simply connected. (The crucial injectivity radius estimate was finished by Cheeger and Gromoll [5] much later for odd dimensional manifolds.) The case when the lower bound 1/4 is included, was settled by Berger [1], in which symmetric spaces of rank one are allowed.

After Milnor [24] found the exotic sphere, it is clear that one wants to prove the manifold is diffeomorphic to the sphere under the same curvature assumption. This is a much more difficult problem. Calabi and D. Gromoll (in his thesis [8]) proved that when $1 / 4$ is replaced by a constant close to one, the manifold is indeed diffeomorphic to the sphere.

The pinching constant was improved in the early seventies by Sugimoto-Shiohama-Karcher [33] (1971), Ruh [29] (1973), Grove-Karcher-Ruh [13] (1974), Hof-Ruh [18] (1975), where the pinching constant was reduced to the order of 0.68. In the mean while, Gromoll and Meyer [9] (1974) found a metric with curvature positive in an open dense set on the Milnor exotic sphere. Recently, Petersen and Wilhelm [27] claimed that they found a metric which has positive curvature everywhere. But this has not been checked thoroughly.

Before Hamilton found his equation, there was no better tools to study the quarter pinching problem for the smooth sphere theorem with optimal constant. A good test 
of the Ricci flow was observed by Hamilton [14] (1982) that if the initial metric is of positive Ricci curvature in dimension three, it will continue to be so under the flow. Then he showed that the flow will converge to a metric with constant curvature, hence the proof of the Poincaré conjecture in this case.

In 1986, Hamilton [15] observed that his flow preserves the positivity of curvature operator in all dimension. Based on this, he proved that four-manifolds with positive curvature operator are diffeomorphic to the sphere or projective space. These were known previously to be homotopic to spheres. In 2008, Böhm and Wilking [2] proved this theorem of Hamilton in all dimensions.

The final settlements of the famous $1 / 4$ pinching smooth sphere theorem was by Brendle and Schoen [3] in 2007, using the Ricci flow. In fact, the pinching condition is replaced by a much weaker pointwise condition: that the ratio of the smallest sectional curvature to the largest one is larger than $1 / 4$ everywhere.

The method used by Brendle and Schoen is rather ingenuous: the $1 / 4$ pinching is not preserved by the Ricci flow and one has to overcome this problem.

First of all, let us discuss the concept of isotropic curvature introduced by Micallef and Moore [23] in 1988 to study the sphere theorem. It comes up in the second variational formula for minimal surfaces as was formulated by Siu-Yau [32] in higher dimensional manifold.

In fact, in 1980, when Siu-Yau [32] proved the Frenkel conjecture that every Kähler manifold with positive bisectional curvature is biholomorphic to complex projective spaces, they minimized the area of a sphere in a fixed homology class. They then proved that the sphere is either a holomorphic or antiholomorphic curve. In doing so, they complexified the normal bundle and solved the CauchyRiemann equations to find a complex holomorphic normal vector field. (Here special features of complex geometry in one dimension is used.) After such holomorphic section is found, the left over terms in the second variational formula was the bisectional curvature when the ambient manifold is Kähler.

When the manifold is Riemannian, the argument that Siu-Yau used still works, except the curvature of the ambient manifold needs to be changed to isotropic curvature which we explain now.

We complexity the tangent space at each point of the manifold, both the metric and the curvature tensor can be extended complex linearly. For any two complex null vectors $u$ and $v$ with zero complex inner product, we require the curvature $R(u, v, \bar{u}, \bar{v})$ in the null plane to be positive. This is called isotropic positivity.

Making use of the second variational formula as was formulated in Siu-Yau [32] in the proof of Frankel conjecture, Micallef and Moore [23] proved that for minimal spheres in manifolds with positive isotropic curvature, the index must be greater than the integral part of $\frac{n-2}{2}$. When the manifold is simply connected, one can use Poincaré duality to prove that simply connected manifolds with positive isotropic curvature must be homotopic spheres. How- ever, this proof cannot tell whether they are diffeomorphic to sphere or not.

Hamilton [15] managed to prove that a simply connected four dimensional manifold with positive isotropic curvature is diffeomorphic to the standard sphere. This was a warm up for his program to prove the Poincaré conjecture in three dimension. Recently, B. Chen, S. Tang and X. Zhu [6] (2008) proved that any four dimensional manifold with positive isotropic curvature is diffeomorphic to the connected sum of $S^{4}$ or $\mathbb{R} P^{4}$ or a quotient of $S^{3} \times \mathbb{R}$.

In 2007, Brendle-Schoen [3] proved that isotropic positivity is preserved under Ricci flow for all dimension (also by Nguyen [25]). In addition to the arguments of Hamilton, they need some delicate algebraic inequalities for curvature tensor with nonnegative isotropic curvature.

Then Brendle-Schoen found a remarkable curvature condition: the isotropic nonnegativity on the product of the manifold $M$ with the two dimensional Euclidean plane, is equivalent to the nonnegativity of $R(u, v, \bar{u}, \bar{v})$ for all $u, v$ on the complexified tangent space of $M$.

They proved that if the strict inequality holds, the Ricci flow will deform the metric to one with constant curvature, after normalization. The pointwise quarter pinching condition on a manifold implies the above condition and hence the proof of the quarter pinching theorem.

There is also rigidity theorem for such statements. Brendle observed that using the above arguments and the holonomy group, one can prove that a simply connected irreducible manifold with nonnegative isotropic curvature is either homeomorphic to the sphere, a Kähler manifold or a symmetric space. For manifolds with pinching slightly less than $1 / 4$, there is work of Peter Peterson and Terry Tao [26] who proved that simply connected manifolds of this type must be diffeomorphic to a sphere or a symmetric space of rank one.

Throughout the entire proof of the smooth quarter pinching sphere theorem, either theory of minimal surfaces are used or Hamilton's equation was used. Both of them required deep geometric analysis. The analysis embedded in the works of Hamilton is very deep; they are not only geometric, but also analytic in nature. A very important gradient estimate was used throughout the proof, It was derived by Hamilton [16] on a tensor inequality modeled after the Li-Yau inequality for heat equation [21].

There are constructions of manifolds with nonnegative sectional curvature based on continuous group actions on manifolds with positive curvature. There is also the double coset construction used by Gromoll and Meyer [9] to construct metrics with positive sectional curvature on an open dense set of the exotic spheres.

Concerning topological obstructions for manifolds with positive sectional curvature, there is a theorem of Gromov [10] who proved that there are upper bounds for their Betti numbers depending only on the dimension of the manifolds. But the bound is far from sharp. The conjecture is that the Betti numbers are not greater than the corresponding Betti number of the torus with the same dimension. 
On the other hand, we know little about the structure of their fundamental group, the ring structure of their cohomology, the Betti number of their loop space, or their homotopic types. In fact, we do not know any manifolds with positive sectional curvature for dimension greater than 24 , which are not symmetric spaces. It would be nice to prove that there are only finite number of diffeomorphic types of such manifolds, each of them should admit some smooth circle group action. Perhaps we may also be reminded of the unsolved Hopf problem on the existence of metrics with positive sectional curvature on the product of spheres.

For complete noncompact manifolds with nonnegative sectional curvature, there is the famous theorem of Gromoll-Meyer-Cheeger-Perelman which says that such manifold is diffeomorphic to a vector bundle over a compact totally geodesic submanifold. This compact manifold is a point if the curvature of the manifold is positive. Hence complete noncompact manifolds with positive sectional curvature are diffeomorphic to the euclidean space (a fact proved by S. Cohn-Vossen for surfaces). It is still an open question to find out which vector bundles over a compact manifold with nonnegative sectional curvature admit a complete metric with nonnegative sectional curvature on their total space.

\section{Kähler Manifolds with Positivity on Curvatures Related to the Complex Structure}

One natural curvature constraint on a Kähler manifold is the positivity of the holomorphic sectional curvature, which is the sectional curvature spanned by any planes spanned by vectors $X$ and $J X$. It is known that the scalar curvature is the average of holomorphic sectional curvature. Hence manifolds with positive holomorphic sectional curvature must have positive scalar curvature and it enjoys the constraints given by such curvature. But there is one more important property that can be derived easily from the second variational formula of geodesics. The fundamental group of such manifolds must be trivial. Since the plurigenera of such manifolds must be trivial, I expect compact manifolds with positive holomorphic sectional curvature are rational manifolds. Conversely, it will be interesting to know which rational manifolds admit Kähler metrics with positive holomorphic sectional curvature. For algebraic surfaces, Hitchin observed that this is always true. But the structure of rational surfaces are rather simple. We probably cannot expect such statements to be true in higher dimension.

In the proof of the Calabi conjecture, a curvature term appeared that is weaker than positive bisectional curvature for Kähler manifolds: For any two complex vectors $V$ and $W$ that are orthogonal to each other, the bisectional curvature determined by them is non negative. This was studied Damin Wu, Fang Yang Zheng, and myself [7] at the beginning and later by Albert Chau and Tam [4]. It turns out that certain $C$ space defined by H. C. Wang satisfy such conditions. It remains a problem to classify such manifolds.

At this point my conjecture that such complete noncompact Kähler manifold are biholomorphic to $\mathbb{C}^{n}$ is still not solved. Progress was made by Shi, X. Zhu, Chen, Chau and Tam, using Kähler Ricci flow.

\section{References}

[1] M. Berger, Sur quelques variétés riemaniennes suffisamment pincées, Bull. Soc. Math. France 88 (1960), 57-71.

[2] C. Böhm and B. Wilking, Manifolds with positive curvature operator are space forms, Ann. of Math. 167 (2008), 1079-1097.

[3] S. Brendle and R. Schoen, Manifolds with 1/4-pinched curvature are space forms, J. Amer. Math. Soc. 22 (2009), 287307.

[4] A. Chau and L.-F. Tam, On quadratic orthogonal bisectional curvature. J. Differential Geom. 92 (2012), no. 2, 187-200. $53 \mathrm{Cxx}$

[5] J. Cheeger and D. Gromoll, On the lower bound for the injectivity radius of 1/4-pinched Riemannian manifolds, J. Differential Geom. 15 (1980), no. 3, 437-442 (1981).

[6] B. Chen, S. Tang, and X. Zhu, Complete classification of compact four-manifolds with positive isotropic curvature, J. Differential Geom. 91 (2012), no. 1, 41-80.

[7] Wu, Damin; Yau, Shing-Tung; Zheng, Fangyang, A degenerate Monge-Ampère equation and the boundary classes of Kähler cones. Math. Res. Lett. 16 (2009), no. 2, 365-374.

[8] D. Gromoll, Differenzierbare Strukturen und Metriken positiver Krümmung auf Sphären, Math. Ann. 164 (1966), 353-371.

[9] D. Gromoll and W. Meyer, An exotic sphere with nonnegative sectional curvature, Ann. of Math. (2) 100 (1974), 401-406.

[10] M. Gromov, Curvature, diameter and Betti numbers, Comment. Math. Helv. 56 (1981), 179-195.

[11] M. Gromov and H. B. Lawson, Spin and scalar curvature in the presence of a fundamental group. I, Ann. of Math. (2) 111 (1980), no. 2, 209-230.

[12] M. Gromov and W. Thurston, Pinching constants for hyperbolic manifolds, Invent. Math. 89 (1987), no. 1, 1-12.

[13] K. Grove, H. Karcher, and E. Ruh, Jacobi fields and Finsler metrics on compact Lie groups with an application to differentiable pinching problems, Math. Ann. 211 (1974), 7-21.

[14] R. Hamilton, Three-manifolds with positive Ricci curvature, J. Diff. Geom. 17 (1982), 255-306.

[15] R. Hamilton, Four-manifolds with positive curvature operator, J. Diff. Geom. 24 (1986), 153-179.

[16] R. Hamilton, The Harnack estimate for the Ricci flow, J. Differential Geom. 37 (1993), no. 1, 225-243.

[17] N. J. Hitchin, Harmonic spinors, Adv. in Math. 14 (1974), 1-55.

[18] H. Im Hof and E. Ruh, An equivariant pinching theorem, Comment. Math. Helv. 50 (1975), no. 3, 389-401.

[19] W. Klingenberg, Über Riemannsche Mannigfaltigkeiten mit positiver Krümmung, Comment. Math. Helv. 35 (1961), 47-54.

[20] H. B. Lawson and S.-T. Yau, Scalar curvature, non-abelian group actions, and the degree of symmetry of exotic spheres, Comment. Math. Helv. 49 (1974), 232-244.

[21] P. Li and S.-T. Yau, On the parabolic kernel of the Schrödinger operator, Acta Math. 156 (1986), 153-201.

[22] A. Lichnerowicz, Spineurs harmoniques, C. R. Acad. Sci. Paris, Série A, 257 (1963), 7-9.

[23] M. Micallef and J. D. Moore, Minimal two-spheres and the topology of manifolds with positive curvature on totally isotropic two-planes, Ann. of Math. 127 (1988), 199-227.

[24] J. Milnor, On manifolds homeomorphic to the 7-sphere, Ann. of Math. (2) 64 (1956), 399-405.

[25] H. Nguyen, Invariant curvature cones and the Ricci flow, $\mathrm{PhD}$ thesis, Australian National University (2007). 
[26] P. Petersen and T. Tao, Classification of almost quarterpinched manifolds, Proc. Amer. Math. Soc. 137 (2009), no. 7, 2437-2440.

[27] P. Petersen and F. Wilhelm, An exotic sphere with positive sectional curvature, arXiv:0805.0812.

[28] H. E. Rauch, A contribution to differential geometry in the large, Ann. of Math. 54 (1951), 38-55.

[29] E. Ruh, Krümmung und differenzierbare Struktur auf Sphären II, Math. Ann. 205 (1973), 113-129.

[30] R. Schoen and S.-T. Yau, On manifolds with positive scalar curvature, course in Berkeley, 1981.

[31] R. Schoen and S.-T. Yau, The existence of a black hole due to condensation of matter, Comm. Math. Phys. 90 (1983), no. 4, 575-579.

[32] Y.-T. Siu and S.-T. Yau, Compact Kähler manifolds of positive bisectional curvature, Invent. Math. 59 (1980), no. 2, 189-204.

[33] M. Sugimoto, K. Shiohama, and H. Karcher, On the differentiable pinching problem, Math. Ann. 195 (1971), 1-16.

[34] H. Yamabe, On a deformation of Riemannian structures on compact manifolds, Osaka Math. J. 12 (1960) 21-37.

[35] R. Schoen, Conformal deformation of a Riemannian metric to constant scalar curvature, J. Differential Geom. 20 (1984), no. 2, 479-495.

[36] Z. Tang, Y. Xie, and W. Yan, Gromov-Lawson-Schoen-Yau theory and isoparametric foliations, arXiv: 1107.5234.

[37] V. A. Toponogov, Riemann spaces with curvature bounded below, Uspekhi Mat. Nauk, 14:185 (1959), 87-130.

[38] V. A. Toponogov, Spaces with straight lines, Am. Math. Soc. transl. 37, 1964.

[39] J. Rosenberg and S. Stolz, Metrics of positive scalar curvature and connections with surgery, Surveys on Surgery Theory, Vol. 2, Annals of Math. Studies, No. 149, Princeton University Press, 2001.

[40] R. Schoen and S. T. Yau, On the structure of manifolds with positive scalar curvature, Manuscripta Math. 28 (1979), 159-183.

[41] M. Gromov and H. B. Lawson, Jr., The classification of simply connected manifolds of positive scalar curvature, Ann. of Math. 111 (1980), 423-434.

[42] M. Gromov and H. B. Lawson, Jr., Positive scalar curvature and the Dirac operator on complete Riemannian manifolds, Publ. Math. I.H.E.S., 58 (1983), 83-196.
[43] Heinz Hopf, Zum Clifford-Kleinschen Raumproblem, Mathematische Annalen (Springer Berlin / Heidelberg) 1926 Vol: 95: 313-339.

[44] Killing, Wilhelm, Über die Clifford-Klein'schen Raumformen, Mathematische Annalen (Springer Berlin / Heidelberg) 1891 Vol: 39: 257-278.

[45] Tian, Gang; Yau, Shing-Tung, Complete Kähler manifolds with zero Ricci curvature. I. J. Amer. Math. Soc. 3 (1990), no. 3, 579-609.

[46] Tian, Gang; Yau, Shing-Tung, Complete Kähler manifolds with zero Ricci curvature. II. Invent. Math. 106 (1991), no. 1, 27-60.

[47] Stolz, Stephan, Simply connected manifolds of positive scalar curvature, Ann. of Math. (2) 136 (1992), no. 3, 511-540.

[48] Marques, Fernando Codá, Deforming three-manifolds with positive scalar curvature, Ann. of Math. (2) 176 (1012), no. 2, 815-863.

[49] Hitchin, Nigel, Harmonic spinors, Advances in Math. 14 (1974), 1-55.

[50] Connes, Alain, Cyclic cohomology and the transverse fundamental class of a foliation, Geometric methods in operator algebras (Kyoto, 1983), 52-144, Pitman Res. Notes Math. Ser., 123, Longman Sci. Tech., Harlow, 1986.

[51] Yau, Shing-Tung, Perspectives on geometric analysis, Surveys in differential geometry. Vol. X, 275-379, Int. Press, Somerville, MA, 2006.

[52] Wang, McKenzie; Ziller, Wolfgang, Einstein metrics on principal torus bundles, J. Differential Geom. 31 (1990), no. 1, 215-248.

[53] Sha, Ji-Ping; Yang, DaGang, Examples of manifolds of positive Ricci curvature, J. Differential Geom. 29 (1989), no. 1, 95-103.

[54] Nash, John C. Positive Ricci curvature on fibre bundles, J. Differential Geom. 14 (1979), no. 2, 241-254.

[55] Mazzeo, Rafe; Pacard, Frank, Maskit combinations of Poincaré-Einstein metrics, Adv. Math. 204 (2006), no. 2, 379-412.

[56] Ruberman, D. Positive scalar curvature, diffeomorphisms and the Seiberg-Witten invariants, Geom. Topol. 5 (2001), 895-924.

[57] Witten, Edward; Yau, S.-T. Connectedness of the boundary in the AdS/CFT correspondence. Adv. Theor. Math. Phys. 3 (1999), no. 6, 1635-1655 (2000). 